\title{
La fiction romanesque comme réceptacle d'anthroponymes chargés de sens chez Alain Mabanckou
}

\section{Romantic fiction as a receptacle for meaningful anthroponyms in Alain Mabanckou}

\author{
Aetius Bassintsa-Bouesso \\ Université Gaston Berger de Saint-Louis du Sénégal \\ aetiusbirahima@yahoo.fr
}

\begin{abstract}
A novel constitutes a fully-fledged universe a product of a writer's imagination. The characters representing some beings move to the core of this universe according to the modes of the fiction. The attributes that permit to reconstitute their portraits are suitable to various interpretations. These not only imply aesthetic considerations (because the writer is first of all an artist), but also sociological and anthropological, insofar the literature would not be dissociated of the realities of the social and communal life. In fact, the name being the identical reference that first specifies the individual and distinguishes him/ her of his/her fellow creatures, it is permissible to see to what point it is rich of implications and "talking" in the novels of Alain Mabanckou. Indeed ; the names of his characters define the people who carry them in the absolute. They suggest aspects of their personality or influence their destiny. And for reason, to seize the senses of which they are invested brings non negligible lightings on the narrations that the congolese writer provides us with.
\end{abstract}

\begin{abstract}
Resumen
Una novela constituye un universo en toda regla, producto de la imaginación de un escritor. En el corazón de este universo se mueven personajes representando a seres humanos según las modalidades de la ficción. Los atributos que permiten reconstruir sus retratos se prestan a diversas interpretaciones. Estas no solamente implican consideraciones estéticas (porque el escritor es "antes de nada" un artista), sino también sociológicas y antropológicas, debido a que la literatura no puede ser disociada de las realidades proprias de la vida social y comunitaria. De hecho, el nombre siendo la referencia identitaria que especifica al individuo a priori, y que lo distingue de sus semejantes, es interesante ver hasta qué punto es rico en implicaciones y "nos habla" en las novelas de Alain Mabanckou. En efecto; los nombres de sus personajes definen totalmente a los seres que los llevan. Sugieren aspectos de su personalidad o incluso influyen en sus destinos. Y con razón, comprender el sentido de sus significados aporta una luz nada despreciable a los relatos del escritor congolés.
\end{abstract}


Anales de Filología Francesa, n. ${ }^{\circ} 28,2020$

LA FICTION ROMANESQUE COMME RÉCEPTACLE D'ANTHROPONYMES CHARGÉS DE SENS CHEZ...

Key-words

Mabanckou, name, character, novel, anthroponym.

\section{Palabras-clave}

Mabanckou, nombre, personajes, novela, antropónimo.

\title{
1. Introduction
}

Alain Mabanckou est né au Congo-Brazzaville en 1966. Il y a passé la quasi-totalité de son enfance et après l'obtention de son diplôme de bachelier du second degré, il entreprend des études supérieures de droit. À 22 ans, il quitte son pays pour la France. "Va, vis et deviens" (Mabanckou, 2013: 25), ce sont les dernières paroles que lui adresse sa mère au moment de leur séparation. Elles resteront inscrites dans sa mémoire comme la bénédiction ultime d'une femme qui l'a élevé du mieux qu'elle a pu, c'est-à-dire avec très peu de ressources, mais qui a su lui accorder toute l'attention que réclame un fils unique. Son exil dans l'Hexagone durera environ quinze ans, pendant lesquelles il aura étudié, puis travaillé comme conseiller juridique chez un grand groupe français. Ses activités d'écriture le conduisent bientôt aux États-Unis, d'abord comme écrivain en résidence à l'Université du Michigan (nous sommes alors en 2002), puis comme professeur de littérature francophone. Ensuite, il est sollicité par la prestigieuse Université de Californie à Los Angeles, où il finit par prendre ses quartiers.

En 2016, Mabanckou devient le premier écrivain à occuper la Chaire de Création Artistique du Collège de France ${ }^{1}$. Pour l'occasion, il présente une leçon inaugurale magistrale qui fera date et le confortera dans son ascension. Par son parcours atypique, sa verve et son goût de la provocation intelligente, il a depuis séduit nombre de lecteurs et sa percée en littérature s'est accompagnée d'un retentissant succès médiatique (Devésa, 2012: 93). Ses romans mettent en scène les aventures cocasses de personnages forts attachants, marqués généralement par un épicurisme bon enfant.

\begin{abstract}
j'ai rencontré l'Imprimeur comme je rencontre souvent la plupart des nouveaux personnages de ce bar, ils sortent de je ne sais où, et les voilà devant moi, les larmes aux yeux, la voix chevrotante [...], moi je jouais à celui qui semblait ne pas s'intéresser à son histoire, les histoires j'en ai entendu, et ce n'est pas d'un seul cahier dont j'aurais besoin pour les rapporter, il me faudrait plusieurs tomes pour parler de ces rois maudits (Mabanckou, 2005: 61).
\end{abstract}

Ces quelques mots extraits de Verre cassé (2005) et prononcés par le personnage éponyme, font écho au contenu de l'ensemble de ses romans. Ces derniers présentent en effet

1 La Chaire de Création Artistique du Collège de France accueille tous les ans des créateurs de différents domaines de l'art. Mabanckou est le premier écrivain à l'avoir occupée. Par sa nomination en 2016, cette institution a offert à l'Afrique noire une plus grande visibilité dans la constellation des littératures de l'espace francophone, comme en témoigne l'intitulé de la leçon inaugurale de l'auteur publiée chez Fayar cette même année: Lettres noires: des ténèbres à la lumière. 
des êtres que la vie n'a pas épargnés: ce sont des "rois maudis". Il y a également à leur sujet quelque chose de l'ordre de l'insaisissable, leurs origines sont inconnues. Ils fourmillent dans la fiction mais "sortent de je ne sais où". Le lecteur doit cependant s'accommoder de leur présence, car "les voilà". Ces êtres de fiction constituent alors le support privilégié du “jeu de prévisibilité qui fonde la lecture romanesque" (Jouve, 1992: 34). Mais qui sont-ils? Répondre à cette interrogation qui n'est pas inopportune, c'est avant tout préciser comment se déclinent leurs identités en les nommant. Notre postulat est que les personnages d'Alain Mabanckou ont une "empreinte" particulière, à mettre précisément au crédit d'une anthroponymie au service de la lisibilité textuelle. Cette constatation nous permet de légitimer l'étude de leurs noms, pour tenter d'en comprendre les fonctionnalités. "L'être du personnage dépend d'abord du nom propre qui, suggérant une individualité, est l'un des instruments les plus efficaces de l'effet de réel", affirmait Vincent Jouve (2006: 59). À travers les noms de ses personnages, Alain Mabanckou délivre des bribes d'informations qui jouent un rôle de premier plan et demandent à leur tour à être "lues" (Pavlovic, 1987: 89). Constituant des attributs essentiels à la désignation et la distinction des protagonistes, ces noms sont tout autant utiles à leur caractérisation. Ainsi souscrivons-nous au jugement par conjecture de Yéo N'gana quand il indique que "noms et personnages sont généralement condamnés à coopérer pour la production du/des sens du texte littéraire, bien que le premier ait longtemps été marginalisé à plusieurs égards par certaines approches critiques" (N'gana, 2017: 1716).

Précisons cependant que l'usage que nous faisons du terme "nom" " ne se limite pas ici aux seuls patronymes. Nous l'étendons délibérément aux prénoms, surnoms ou sobriquets, pourvu qu'ils soient évocateurs et que leurs contenus sémantiques se prêtent à des commentaires édifiants. Il est nécessaire que nous levions l'équivoque sur ce point, de peur que nous nous attirions le reproche d'utiliser ce terme sans aucune rigueur euristique, dans l'usure sémantique et nominaliste que la mode fait encourir à toute notion trop usitée.

Cette contribution s'appuiera sur un corpus composé de cinq ouvrages: African psycho (2003), Les petits-fils nègres de Vercingétorix (2002), Verre cassé (2005), Mémoires de porc-épic (2006) et Tais-toi et meurs (2012). Elle ambitionne de montrer que l'écrivain congolais nomme ses personnages en fonction de considérations précises. Il s'agit tantôt de renseigner le lecteur sur l'orientation des récits dont ils sont les acteurs, tantôt d'élucider des réalités congolaises puisque le Congo est l'univers de référence de son imaginaire (Mabanckou, 2012: 131). Dans d'autres cas d'espèce, nous verrons que l'intelligibilité des anthroponymes répertoriés dans les romans de Mabanckou tient à leur expressivité en français, notamment quand ils sont articulés sur une imagerie qui convoque un certain symbolisme.

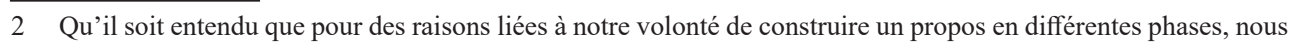
considérerons comme nom tout anthroponyme, si farfelu soit-il, constituant l'appellatif d'un personnage mentionné dans le corpus. Ce n'est qu'à condition qu'il vienne en complément d'un autre anthroponyme connu que nous le classerons parmi les surnoms. 


\section{Les noms issus des langues du Congo}

Conçus dans le cadre de la fiction, les personnages de Mabanckou, par les noms qu'ils portent, sortent de l'anonymat, même quand le romancier tente de faire leurs portraits au moyen d'une écriture plus conventionnelle. Leurs noms, inspirés des langues du Congo, sont des données loin d'être dénuées de significations. Ils participent à leur mise en système, comme l'exemplifie le patronyme de Mpassi-Mpassi dans Les petits-fils nègres de Vercingétorix. En effet, en kikongo ${ }^{3}$, le terme mpassi constitue une unité lexicale autonome faisant référence à la “souffrance". La compréhension de l'usage que l'auteur fait de ce terme requiert toutefois la prise en compte de la manière dont se trouve organisée le récit. Dans ce roman, Mpassi-Mpassi n'est pas fondamentalement sujet à des afflictions, mais il est celui qui cause des angoisses et tourmente les membres de sa famille en raison de ses inconséquences. Son patronyme en dit alors long sur sa conduite, car il laisse présager de la souffrance psychologique résultant des sentiments d'exaspération et d'impuissance ressentis par des parents face à un enfant incorrigible. Son mode de construction, par le redoublement de cette unité lexicale empruntée au kikongo, s'ouvre à des connotations hyperboliques suggérant les travers du personnage, et cette intention est communiquée par l'entremise d'un discours de clarification tenu par la narratrice:

Il n'y avait pas une seule semaine où quelqu'un de Batalébé ne venait se plaindre de son comportement. Le vol des fruits pouvait, à la rigueur être absent. Mais avec les bandes de gamins du centre de Batalébé, ils s'en prenaient (Mpassi-Mpassi et les fameux gamins) aux moutons, aux porcs et à la volaille des habitants. Le père, qui aurait pu redresser cette déviance, avait rejoint l'autre monde (Mabanckou, 2002: 80).

Mpassi-Mpassi c'est donc l'enfant turbulent et impénitent. Décrié par tous, il persiste dans ce qui est moralement répréhensible. Jamais il ne fait preuve de bon sens, jamais il n'entend la voix de la raison. Entraîné par ses camarades dans les grandes villes du pays dès l'âge de quinze ans, il s'adonne à la consommation de drogues et bascule dans la délinquance, au grand dam de sa mère et de sa sœur Christiane. Les soucis qu'il leur cause sont d'autant plus grands que devenu adulte, Mpassi-Mpassi quitte son pays pour l'Europe et ne donnera plus aucun signe de vie.

Dans Les petits-fils nègres de Vercingétorix, Mabanckou traite également du problème des antagonismes entre les ethnies du nord et du sud du Congo, qui ont engendré les guerres que ce pays a connues en 1997 et 1998 (Ngoïe-Ngalla, 1999). Il ne faut pas s'y tromper, c'est évidemment le Congo qui se mue en Viétongo dans la fiction romanesque, par le truchement de la transposition. Ces conflits avaient débuté en milieu urbain avant de s'étendre dans l'arrière-pays, par la faute de politiciens véreux, désireux de se tailler un fief dans leurs villages

3 Le kikongo est la deuxième langue nationale du Congo. Il est très usité dans le sud du pays, notamment à PointeNoire, la capitale économique où est né Alain Mabanckou. 
respectifs. Schéma clairement reproduit dans le roman. Nordistes et sudistes s'entretuaient et toute collaboration avec l'ethnie adverse était perçue comme une trahison. Partant de cette considération, Ossouki Wapi est un personnage dont le nom nous interpelle. En lingala ${ }^{4}$, cet accolement notionnel équivaut à une interrogation oratoire qu'on adresse, dans le parler courant et avec un ton sarcastique, à un individu à qui l'on reproche certains faits dont il paie le tribut. Il participe d'un acte du langage à fonction illocutoire, traduisible en français par "où cela t'a-t-il mené?". Dans le roman, Ossouki Wapi est un homme originaire du nord du Viétongo, mais qui a été ministre dans le gouvernement d'un régime sudiste. Le général Édou, natif du nord comme lui, se félicitera de pouvoir l'humilier publiquement en raison de son “infidélité aux siens" (Mabanckou, 2002: 178). Dans le cas d'espèce, le nom du personnage est révélateur du traitement qui lui sera réservé et du désaveu dont il a fait l'objet de la part de sa communauté pour avoir servi les intérêts ennemis.

En outre, dans Verre cassé, Lokouta Éleki Mingi est le patronyme d'un dirigent odieux, tandis que Tembé Essouka, dans Mémoires de porc-épic, est le nom donné à un féticheur de renom. En scrutant ces deux dénominations sur la base d'une approche linguistique purement traductive, il apparaît que la première constitue un appellatif correspondant à la phrase minimale "il ment comme il respire". La seconde, elle, signifie littéralement "que le doute soit dissipé". Qu'est-ce que cela implique dans l'ordre de la posture énonciative de l'auteur? Pour le comprendre, il faut tenir compte des modes de représentation du monde proposés par ses romans. Lokouta Éleki Mingi est un personnage qui sert de support à une écriture mettant en accusation la figure de l'homme politique qui, entre promesses électorales irréalisables et discours oiseux, sombre pitoyablement dans la mythomanie. Le contenu sémantique du nom de ce personnage signale cet aspect de sa personnalité qu'Alain Mabanckou s'emploie à dépeindre de façon caricaturale. Pour ce qui est de Tembé Essouka, les doutes des incrédules sur son art de la divination doivent en effet s'estomper puisqu'il se montre toujours capable d'interroger le passé, de prédire l'avenir ou de lever le mystère sur des choses qui échappent au sens commun (Bassintsa-Bouesso, 2018: 62). Chez Alain Mabanckou, les noms des personnages construits à partir des langues du Congo ont donc une fonction explicative. Ce sont des indicateurs qui donnent différents repères utiles à la compréhension des rôles qui leur sont dévolus dans l'intrigue.

Ce code anthroponymique qui consacre les langues congolaises, "en plus de révéler le(s) personnage(s), [aide] à tisser la structure de la narration. [Il fait des noms des protagonistes] des récits intradiégétiques [à part entière] qui unissent, fondent et confondent univers fictionnel et réalité" (N'gana, 2016: 1716). Alain Mabanckou l'utilise comme un instrument par lequel il dessine les scénarios de ses romans. Élaborés selon une forme de pensée dynamique les noms propres sont dès lors érigés en "moyen de communication, de positionnement

4 Le lingala est la première langue nationale du Congo. Parlé à Brazzaville et au nord du pays, c'est une langue que le Congo - Brazzaville a en commun avec la République Démocratique du Congo (R.D.C) et qui facilite les échanges entre les deux pays. 
et de divulgation idéologiques" (N'gana, 2016: 1717). Ce parti pris méthodologique n'est pas en soi une nouveauté chez les auteurs originaires du continent africain. C'est un exercice auquel se sont livrés plusieurs d'entre eux comme le fait remarquer Bosson Bra lorsqu'il dit: "le choix des noms des personnages [...] obéit à des déterminations dans les néologismes du terroir; et l'acte de dénomination devient un acte de naissance, de reconnaissance et d'identité" (Bra, 2013: 103). Nous retrouvons par exemple les traces d'un pareil agenda chez Henri Lopes dans Le pleurer-rire (1982) et chez Alphonse Mayimona dans Le retour triomphal de Mossi (1992). Que faut-il en effet attendre d'une femme nommée Soukali5', si ce n'est qu'elle sache se faire désirer et qu'elle soit réputée pour ses performances sexuelles? Dans le même sillage, Mossi ${ }^{6}$ est le fils unique tant aimé malgré sa prodigalité, comme l'insinue son nom.

Il est donc évident que les potentialités référentielles des anthroponymes construits à partir des langues congolaises donnent aux textes du corpus une certaine souplesse. L'auteur parvient à surdéterminer ses personnages et à circonscrire leurs comportements par un système de nomination que l'énonciation se charge de valider. Dans cette perspective, certains de ses personnages portent les marques d'un projet global à travers leurs noms. Si Grégoire Nakobomayo travaille à perpétrer un crime dans African Psycho, son patronyme exprime à suffisance le forfait qu'il entend commettre. Et pour cause, Nakobomayo est la forme prise au futur par le verbe lingala koboma (tuer). Lorsque celui-ci est associé aux pronoms na (moi / je) et yo (toi/ tu) par des processus de préfixation et suffixation, cela donne en un mot “je-te-tuerai”. Dans African psycho, cet individu névrosé est justement obsédé par l'idée d'assassiner une prostituée qui s'appelle Germaine. L'incipit du roman, se veut d'ailleurs à cet effet informatif, car il embraie fort bien le programme narratif cristallisé sur ce projet: “J'ai décidé de tuer Germaine le 29 décembre. J'y songe depuis des semaines parce que, quoi qu'on dise, tuer une personne nécessite une préparation à la fois psychologique et matérielle" (Mabanckou, 2003: 11). En procédant de la sorte, Alain Mabanckou instaure une cohérence entre l'action romanesque et le personnage qui est programmé pour l'accomplir. Ce qui nous amène à conforter Roland Barthes dans ses propos quand il soutient: "le nom propre est lui aussi un signe, et non bien entendu une simple idée qui désignerait sans signifier [...]. Comme signe, [il] s'offre à une exploitation, à un déchiffrement” (Barthes, 1972: 125).

Dans Tais-toi et meurs, nous notons que les noms congolais influencent aussi la vie des personnages et résument parfois leur existence. Julien, dont le patronyme "Makambo" veut dire "les ennuis", se trouve mêlé malgré lui à une histoire qui vire au drame avec la mort d'une jeune femme par défenestration. Bien qu'innocent, il va finir en prison et sera le bouc-émissaire qui endossera à ses dépens la responsabilité de l'affaire. "Makambo" est un

5 Soukali est un personnage d'Henri Lopes dont le nom veut dire "sucré(e)" ou "succulent(e)" en lingala. Dans Le pleurer-rire, Soukali est décrite comme une femme d'une beauté à couper le souffle et très active sexuellement, au grand bonheur de ses amants. Elle a des formes généreuses et son pouvoir de séduction lui permet de multiplier ses conquêtes.

6 Mossi est le nom d'un personnage d'Alphonse Mayimona. Ce nom désigne en kikongo le chiffre "un (1)". Dans le contexte du roman de cet écrivain, il fait référence au statut de fils unique qu'a le personnage qui le porte. 
nom que ce personnage considère lui-même comme étant de mauvais augure, tant il semble lui porter malheur. Sa vie toute entière, justement faite d'ennuis, rime parfaitement avec celui-ci:

Je m'appelle Julien Makambo [...]. Dans notre langue du Congo-Brazzaville, le lingala, Makambo signifie les ennuis. J'ignore ce qui avait piqué mes parents pour m'attribuer un tel nom, qui n'est d'ailleurs pas celui de mon défunt père, encore moins celui d'un proche de la famille. Je suis maintenant convaincu que le nom qu'on porte a une incidence sur notre destin (Mabanckou, 2012: 9).

Une œuvre romanesque est naturellement organisée autour de personnages à étudier en fonction des relations actantielles qui sont opérantes dans le texte. Chez Mabanckou, ces relations appellent une sémiologie que les noms laissent transparaître en filigrane. Ces derniers constituent, pour ainsi dire, des interfaces qui déterminent les interactions entre les protagonistes. Dans le même temps, ils sont associés à une stratégie qui en fait des données intégrant une démarche de singularisation des personnages, en élucidant la partition de chacun d'entre eux dans l'orchestration événementielles du récit. L'auteur fait de telle sorte que les noms, puisés dans le lexique du lingala et du kikongo, soient en adéquation parfaite avec les aventures des protagonistes, qu'ils traduisent de manière concrète des faits légitimant les récits dont ils sont les acteurs. Ses romans corroborent par conséquent une acception anthropologique de l'acte de nomination notable dans nombre de sociétés africaines considérant, comme le dit Jacques Fédry, que "le nom c'est l'homme" (Fédry, 2009). Quand Julien Makambo est recherché par la police, il décide de quitter Paris pour Nantes afin d'échapper à une arrestation. Il rencontre un homme nommé Kofunda ${ }^{7}$ qui va l'épier, puis fournir des informations le concernant à Shaft, individu controversé qui le livrera. Kofunda est celui qui a trahi Julien et c'est bien ce à quoi fait référence son nom.

Par ailleurs, remarquons avec Abraham Ndinga-Mbo que sur la mosaïque des groupes ethniques qui recouvrent le territoire du Congo-Brazzaville, les disparités linguistiques donnent non seulement l'impression que les Congolais sont une population hétérogène, mais aussi que les particularités des cultures régionales font des noms une "étiquette" qui renseigne sur l'origine ethnique de l'individu (Ndinga-Mbo, 1998: 40-49). Cette réalité sociologique, inhérente au milieu dans lequel Alain Mabanckou a grandi, prend forme dans ses romans.

Si les préfixations en "I" et en "O" sont fréquentes dans la construction des patronymes au nord du Congo, en particulier chez les Mbochi ${ }^{8}$, Hortense Iloki et Gaston Okemba, personnages de la fiction romanesque dans Les petits-fils nègres de Vercingétorix, sont également présentés comme des natifs du nord. Dans le même ordre d'idées, Kimbembé, Mam'

7 Kofunda signifie "trahir" en lingala.

8 Les Mbochi, encore appelés Bambochi, sont une population d'Afrique centrale établie au nord du Congo, précisément dans les départements de la Cuvette et des Plateaux. 
Soko, Youla, Massengo, Kengué, sont des noms à forte résonnance sudiste, très répandus chez les Kongo ${ }^{9}$ et les Beembe ${ }^{10}$, et les personnages qui les portent dans les romans à l'étude s'identifient à ces groupes. Par conséquent, chez Alain Mabanckou les noms issus des langues du Congo sont aussi des repères identitaires dévoilant la provenance géographique et l'appartenance ethnique. Ils s'emploient à donner une teinte réaliste à ses romans quand ils tentent de reproduire les clivages régionalistes encore patents dans son pays. L'auteur “joue sur les connotations socio-tribalistes qui emplissent la société représentée [dans ses romans] pour donner à voir ce qui fonde les oppositions entre différentes communautés" (Taba Odounga, 2013: 89).

Ce mode de conception des noms propres a des implications narratives évidentes et repose sur des choix faits dans l'optique d'une mise en perspective des personnages. Pour l'auteur, convoquer les langues congolaises dans l'attribution des noms c'est établir des points d'ancrage qui portent à la connaissance du lecteur les rôles dans lesquels il entend "enfermer" les protagonistes de ses récits. Ces noms ne sont donc pas donnés de façon aléatoire. Ils servent parfois à dessiner les contours d'un caractère qui se confirme au fur et à mesure que s'enchevêtrent les événements de l'intrigue. Mais le lecteur qui ne partage pas les mêmes codes linguistiques que lui est quelque peu frappé d'une cécité partielle édulcorant l'acuité de sa réception. Tous ces noms congolais peuvent lui paraître étranges, cacophoniques, voire fantaisistes. Mais dans un exercice de compensation de leur inintelligibilité pour ce type de public, Alain Mabanckou élargit leur répertoire à de termes dont les effets de sens particuliers sont susceptibles d'être saisis par tous les francophones.

\section{Les noms expressifs en français}

La langue française est pour beaucoup d'écrivains africains, soucieux de s'inscrire dans la constellation de la world literature ${ }^{11}$, un support communicationnel favorisant une large diffusion de leurs textes. C'est dire que le français comme langue d'écriture, faut-il le rappeler, n'est pas l'apanage des auteurs de l'Hexagone. D'autres écrivains l'utilisant ont enrichi la littérature par leur double appartenance culturelle et leur génie (Gasquy-Resch 200: 9). Le débat sur les littératures africaines qui, du point de vue de certains intellectuels, devraient être produites dans les langues du continent, semble en conséquence ne plus s'inscrire dans l'air du temps (Kesteloot, 2004: 209-213). Nous sommes d'avis, comme Rainier Grut-

9 Les Kongo, encore appelés Bakongo, sont une population bantoue occupant le sud du Congo, en l'occurrence les départements du Pool, de la Bouenza, du Niari, de la Lékoumou et du Kouilou. Ils sont aussi présents dans l'ex-Zaïre, mais encore dans le nord de l'Angola où ils établirent la capitale d'un puissant et vaste empire dans l'antiquité: Mbanza-Kongo.

10 Les Beembe, ou Babeembe, constituent un sous-groupe du grand ensemble Kongo. Si nous les dissocions dans notre étude, c'est uniquement par commodité avec la fiction romanesque car, dans Les petits fils nègres de Vercingétorix, un personnage comme Mam' Soko s'identifie comme une Beembe et non comme une Kongo.

11 Concept anglo-saxon qui restreint la notion de "mondialisation" au champ de la littérature. 
man, que cette problématique se pose désormais en des termes nouveaux (Grutman, 2002: 72-73). L'histoire coloniale a marqué les auteurs africains de la première heure qui avaient un rapport conflictuel à la langue française, parce qu'elle leur avait été imposée au détriment de leurs langues maternelles. Mais avec les écrivains de la nouvelle génération, vivant pour la plupart en expatriation dans un monde que l'on dit "globalisé", le multilinguisme est désormais saisi comme un atout permettant de dire sa pluralité. Nous relevons que l'œuvre d'Alain Mabanckou est le lieu d'expression d'un rapport dialectique, voire dialogique, entre les langues congolaises et le français. L'usage du lingala et du kikongo dans l'affectation de noms à ses personnages est, nous l'avons vu, la manifestation d'un sentiment d'attachement à sa terre natale, mais par ailleurs le français reste sa principale langue d'écriture. Il le met alors à contribution dans son approche créatrice qui fait du nom un maillon essentiel de la chaîne des attributs de ses personnages.

Dans les romans à l'étude, les anthroponymes sont donc aussi conçus de manière à permettre au lecteur francophone d'augurer de leur compatibilité avec le référent textuel lié aux personnages qu'ils désignent. Mais qu'est-ce qu'un nom en fin de compte?, s'interroge d'ailleurs l'auteur lui-même, avant de noter dans son essai intitulé Lettre à Jimmy: presque rien et tout à la fois. On le porte avec fierté quand on peut le rattacher à un passé glorieux ou à des faits gratifiants, mais il devient humiliant s'il évoque par exemple l'illégitimité (Mabanckou, 2007: 13).

Nous observons dans le corpus que les noms expressifs en français sont tributaires d'une compétence descriptive les justifiant, en ce sens qu'ils se voient délégués la mission de rendre compréhensibles les axes thématiques ayant servi à nouer les intrigues. Dans Verre cassé par exemple, Zéro-Faute est censé ne jamais se tromper dans ses diagnostics et ses prescriptions. Quand des clients viennent consulter ce féticheur à la réputation sulfureuse, il tient un discours d'autoglorification dans lequel il s'attèle à garantir le succès de ses opérations incantatoires à venir, en tâchant de faire de son nom le relai de son expertise en matière d'exorcisme: “comprenez bien que c'est le diable qui habite le corps de votre époux, je vous promets que nous allons sortir ce diable de son corps, croyez-moi, je ne m'appelle pas Zéro-Faute par hasard, et comme vous le savez tous, j'ai lutté contre des esprits bien plus rebelles" (Mabanckou, 2005: 167). Dans la même optique, on s'accommodera du fait que le nom de Robinette soit conçu, par dérivation, à partir de la base nominale "robinet", puisque dans l'épisode pittoresque où la femme qui s'appelle ainsi fait son apparition, "elle met au moins dix minutes à uriner sans s'arrêter, ça coule et coule encore comme si on avait ouvert une fontaine publique" (Mabanckou, 2005: 94).

Nous conviendrons également que L'Escargot-entêté est un nom qui suggère le corps frêle du personnage qui le porte d'une part, puis l'obstination et l'abnégation dont il a fait preuve avant de connaître le succès dans les affaires d'autre part. Lorsque ce personnage décide de débuter une activité commerciale en ouvrant un bar en effet, nombreuses sont les voix 
qui s'élèvent pour s'y opposer. Mais en dépit de toutes sortes de menaces et de l'adversité de ses détracteurs les plus farouches, personne ne parvient à l'en dissuader:

on lui avait promis qu'il mangerait la pomme de l'eau, qu'il deviendrait un clochard, un bout de bois de Dieu, un damné de la terre, qu'il dormirait dans les tonneaux comme certains philosophes du passé, et pourtant L'Escargot-entêté est toujours là, et pourtant il est toujours debout, résolu comme un joueur d'échecs, et il a vu les années passer en un combat douteux, et il a vu les jaloux se lasser de lui chercher des poux [...]. L'Escargot-entêté avait résisté, il s'était réfugié dans sa bave de gastéropode endurci (Mabanckou, 2005: 37-38).

S'agissant de Verre cassé, héros du roman le plus connu de Mabanckou, deux explications nous paraissent plausibles: le substantif "Verre" est révélateur de ce que ce personnage est un sujet ayant un goût immodéré pour les boissons enivrantes. L'élément adjectival "cassé”, auquel il est associé, laisse préfigurer sa mort prochaine car Verre cassé se suicidera. Il trouvera la mort après qu'il se sera jeté dans la rivière Tchinouka. Mais étant donné que dans la culture populaire des éclopés dépeints dans le roman, les adeptes des lieux de réjouissance que sont les $N g a n d a^{12}$, considèrent qu'un ivrogne "digne de ce nom" devrait boire nom pas dans un verre mais directement en bouteille, on peut s'imaginer dans un second temps que le "Verre" est "cassé" parce que le personnage de la sorte nommé, désireux de s'affirmer comme un ivrogne sans égal, n'en fait que rarement usage. Quoi qu'il en soit, ce nom permet au lecteur francophone d'établir de façon spontanée et intuitive le rapport addictif à l'alcool de ce personnage.

Les anthroponymes expressifs en français apportent dans ce sens des éclairages considérables et dans leur fonctionnement mimétique, ils sonnent justes. Par eux la fiction romanesque acquiert une sorte de crédibilité et ils permettent de renforcer l'effet de congruence recherché par l'auteur. Mabanckou affiche la volonté de faire en sorte qu'ils s'ouvrent à des évocations précises. Les modalités de dénomination qu'il met en place visent à accompagner ses personnages dans l'acquisition d'une identité. C'est là une constante de ses romans. Elles dévoilent des défauts, des tares, des ambitions, des volontés, etc. On peut toujours s'attendre à en apprendre davantage sur ses personnages grâce à leurs noms. En se mettant au service de la régulation de la mécanique du dispositif romanesque, ces derniers donnent des pistes quant à son orientation. Cela permet au lecteur d'émettre des hypothèses les concernant, hypothèses qui se vérifient au fil du déroulé de l'histoire.

Parfois encore, ils s'inscrivent dans la logique d'une écriture faisant la part belle à la dérision et la raillerie. Dans Tais-toi et meurs par exemple, quand le narrateur raconte les tribulations d'un jeune immigré à Paris nommé Bonaventure, il mentionne ses conditions de vie misérables et la précarité des emplois qu'il a occupés, puis il formule la remarque que

12 Le mot ngada sert à désigner les bars et autres débits de boisson populaires au Congo. Il est d'usage tant en lingala qu'en kikongo. 
voici à son sujet: "avec un tel prénom (Bonaventure), je ne sais pas s'il avait été gâté par la vie puisque rien dans son existence n'avait montré que son aventure avait réussi en France" (Mabanckou, 2012: 44).

De même, Prosper, autre protagoniste du même roman, a toujours été dépendant de Pedro et Moussavou, alors que son prénom et la qualification louangeuse "prospère", renvoyant à l'idée du progrès et désignant un état d'opulence, se prononcent à l'identique. À cette occurrence s'ajoute Angélique, le prénom de la femme de Verre cassé. S'il est référencé comme une épithète qualifiant "ce qui est digne d'un ange", Verre cassé décrit plutôt sa femme comme possessive et autoritaire. Il la surnommera d'ailleurs parodiquement "Diabolique". Ce prénom, dont la signification est en principe associée à l'idée du divin (l'ange étant en effet un être céleste), contraste avec le profil psychologique de la femme qui le porte. Dès lors, le lecteur francophone est tenu d'apprécier les noms des personnages d'Alain Mabanckou comme des morphèmes ouverts à des contre-sens, étant donné que les portraits que l'auteur brosse de ses personnages se situent parfois aux antipodes des significations que la langue française leur prête. En de telles circonstances, ils viennent agrémenter le souffle ironique dont le style du romancier est imprégné. Les textes d'Alain Mabanckou, en effet, "reposent sur des narrations qui créent délibérément des écarts entre les attentes nourries par le lecteur et les scènes qui s'imposent à lui, entre les discours tenus par les personnages et les situations qui les induisent" (Bassintsa-Bouesso 2018: 68). Les noms de cette catégorie deviennent des artifices qui se déploient dans l'élan du traitement ironique qu'il fait de la langue française, par le biais d'un jeu sémantique de contraires posant le défi de la compréhension de la fonction évaluative d'une anthroponymie axée sur des idées prises à revers, ayant partie liée avec l'exploitation de ce procédé (l'ironie) à des fins esthétiques. (Taba Odounga, 2013: 80).

\section{Le symbolisme des surnoms ou sobriquets}

À défaut de se voir attribuer des sobriquets par leurs compères, les protagonistes du corpus à l'étude choisissent volontairement d'en porter. Ces appellations qui prennent le pas sur leurs vrais noms rendent compte des flux et reflux de leur psychologie. Par le jeu du détour, elles évoquent tantôt des scènes de vie et des expériences partagées, tantôt des déviances ou des a priori que ces personnages inspirent à autrui. Ces noms de substitution les marquent d'un sceau spécifique et les rendent moins "opaques". Mais encore faut-il en comprendre les mobiles!

Comme le précise Antonio Marques (2015), les sociétés humaines sont régies depuis toujours par un principe général qui veut que les individus aient un nom. Cependant, le besoin d'y ajouter quelque chose de différent se fait parfois sentir. Le recours au sobriquet aide alors à ajouter au nom un élément nouveau en accord avec des distinctions physiques, des 
traits de caractère et parfois même en lien avec une réputation. Même si cette dernière ne repose que sur des préjugés. Ainsi, dans Verre cassé, les filles de nationalité camerounaise qui résident à Paris sont par exemple surnommées les "cameruineuses" dans les cercles de la diaspora africaine, au motif qu'elles ne seraient que des arrivistes (Mabanckou, 2005: 140). Les personnalités publiques n'échappent pas, elles non plus, au port de sobriquets et Marie Treps (2012) en fait le constat dans ses travaux. Il s'agit là d'un phénomène populaire avéré et Mabanckou l'inscrit volontiers dans son œuvre. Dans Les petits-fils nègres de Vercingétorix, le politicien Ta Kanda se fait appeler "Vercingétorix", parce que inspiré par la figure de la résistance gauloise contre les velléités colonialistes de Rome qui se nommait ainsi (Porte, 2013). Il choisit en effet ce sobriquet en se comparant au personnage historique, car lui aussi est un chef rebelle qui prétend combattre le régime en place au Vietongo au nom des siens. Le choix de ce surnom est donc fait en fonction d'une situation politique qui prévaut. Il est l'expression d'une volonté du personnage concerné d'augmenter son charisme et sa réputation, en s'identifiant à ce guerrier dont l'histoire a retenu la capacité de fédérer les peuples gaulois pour faire front contre Jules César.

Répondre au surnom "Vercingétorix" le fortifie dans la conduite de sa rébellion. Par ce sobriquet se manifeste un désir de détourner l'écho retentissant de la gloire passée du vrai Vercingétorix afin de se construire une subjectivité individuelle. Il se voit en effet dans la peau de l'irréductible gaulois en tenant tête à ses ennemis, mais dans le contexte d'un conflit armé propre au Viétongo (Mabanckou, 2002: 200-201).

Motivés par un ethnocentrisme béat, les miliciens qui lui sont fidèles sont surnommés ses "petits-fils nègres". Le vocable que recouvre ce syntagme nominal met en exergue leur dévouement sans bornes à leur chef et véhicule à n'en point douter l'idée d'un lien indissoluble qui les unit à lui, par analogie à celui qu'un aïeul entretient avec sa descendance. Vercingétorix est pour eux, en effet, bien plus qu'un guide éclairé. Ils le considèrent comme un patriarche dont la clairvoyance supposée mènerait tous les peuples du sud du Viétongo à bon port en combattant le général Édou. Ceci explique la dénomination première du chef rebelle (Ta Kanda) qui, en kikongo, veut dire "celui qui est de la famille" et suggère un lien de parenté.

Comme l'indique Antonio Marques, Il ne peut y avoir de sobriquet sans relation de causalité. Les sobriquets sont toujours ajustés selon des circonstances déterminées, et il est de bon aloi d'en remarquer la diversité des sources chez Mabanckou. "Ils composent un univers bigarré, mouvant, kaléidoscopique, un univers d'illusion parfaite, d'étonnement perpétuel et de fugacité absolue" (Pavlovic, 1987: 89). Il arrive qu'ils soient puisés dans le bestiaire de manière à calquer une image animalière sur les personnages. À titre illustratif, Shaft dans Tais-toi et meurs est surnommé "Le Caméléon", vu son aptitude à contourner toutes les dispositions prises par les autorités, sa capacité à s'adapter à toutes les situations pour pérenniser ses activités de faussaire (Mabanckou, 2012: 60). Chinua Achebé dans Le monde 
s'effondre avait lui aussi donné à son personnage Amalinze un sobriquet du même ordre: "Le chat". Resté invaincu pendant de longues années, on disait de ce lutteur que personne ne parviendrait à le terrasser, que son dos ne toucherait jamais le sol à l'image du félin du même nom qui retombe toujours sur ses pattes (Achebe, 2000: 9).

Antonio Marques explique aussi, dans ses travaux sur les sobriquets en littérature, que ces derniers servent encore à superposer le "je" social au "moi", quand un écrivain produit une œuvre socialement engagée. Il précise que l'œuvre socialement engagée doit nécessairement donner la parole aux couches populaires pour lesquelles le recours aux sobriquets est plus que tout un procédé générateur d'un enthousiasme personnel ou collectif. Le moins que l'on puisse dire, c'est que Mabanckou admet son appartenance à cette catégorie d'écrivains, ce qui le conduit à souvent réexaminer ses personnages:

Dans mes premiers écrits (tous ébauchés au Congo), je sentais qu'il manquait des pièces, que mes personnages étaient cloîtrés, respiraient à peine et me réclamaient plus d'espace. L'émigration a contribué à renforcer en moi cette inquiétude qui fonde à mes yeux toute démarche de création. On écrit parce que quelque chose ne tourne pas rond, parce qu'on veut déplacer les montagnes ou faire passer un éléphant dans le chas d'une aiguille. L'écriture devient alors à la fois un enracinement, un appel dans la nuit et une oreille tendue vers l'horizon (Mabanckou, 2012: 132).

Chez les petites gens, le sobriquet, quand il est magnifiant, s'exhibe avec fierté, car il élève l'individu au-dessus de sa condition réelle et lui offre une certaine visibilité au sein de sa communauté. Par lui se manifeste une entreprise de dévoilement de soi sous de nouveaux auspices pour tenter d'aboutir à la construction d'un nouvel ethos. Cela se vérifie dans les textes à l'étude avec un personnage comme Pedro qui se fait appeler "Le Maire de Paris" (Mabanckou, 2012: 49). Pedro a une parfaite connaissance des rouages de tout un réseau parisien de trafiquants de fausse monnaie et de faux papiers. Il a d'importants contacts, haut placés dans la hiérarchie de ces malfaiteurs et sa parfaite connaissance de la ville lui permet d'échapper aux traques policières. Dans cet univers, son sobriquet, tablant sur un détournement tropique par antonomase, entre en résonnance avec sa connaissance de tout un système. Il lui assure le succès auprès de la communauté congolaise de la capitale française, dans laquelle il y a beaucoup de sans papiers voyant en lui une personne-ressource essentielle, par analogie à ce que représente l'élu pour ses administrés.

Nous distinguons enfin, dans les romans à l'étude, des sobriquets qu'il conviendrait de qualifier de "taquins". Dans les rapports qu'ils entretiennent, les personnages d'Alain Mabanckou en usent pour moquer leurs compères, mais sans réelle méchanceté. Ces sobriquets, connus d'un groupe restreint d'individus liés d'amitié, renforcent leur complicité et insufflent à leurs relations une dimension émotionnelle forte. L'illustration la plus probante que nous puissions donner en la matière est celle de Mama Mfoa dans Verre cassé. Femme aimable et aimante, elle vend des brochettes de viande devant le bar Le Crédit a Voyagé. Elle 
est la seule personne du quartier à porter sur les ivrognes qui fréquentent ce débit de boisson, notamment sur Verre cassé, un regard tendre et affectueux: elle lui donne des brochettes à crédit, se soucie de sa santé, lui prodigues des conseils, etc. Et le héros nous la dépeint en disant: "elle est chauve et chante de temps à autre pour nous amuser, c'est pour cela qu'on l'appelle affectueusement La Cantatrice Chauve" (Mabanckou, 2005: 149). Il ne nous aura pas échappé que ce surnom taquin, qui participe du jeu anthroponymique dont les romans de l'auteur sont le réceptacle, s'appuie concomitamment sur le procédé de l'intertextualité, puisqu'il est emprunté au titre d'une pièce à succès d'Eugène Ionesco.

Les sobriquets repérables dans les romans à l'étude fixent en majorité un périmètre de reconnaissance favorisant des rapprochements de leur sens littéral avec le substrat qu'ils désignent. Cela permet à l'auteur de rendre tangible la fécondité de son imagination et de jouer doublement sur la notion d'identité. Ils sont à cet égard un marqueur d'identité en ce qu'ils disent qui sont les personnages (ils permettent de les identifier), mais encore parce qu'ils conditionnent cette identification à la compréhension d'une identité (au sens de similitude) avérée entre ceux-ci et des réalités substantielles avec lesquelles ils ont une nature commune. Alain Mabanckou convoque ainsi leur fonction iconique, créatrice de sens. Sens qui se veut univoque, laissant entrevoir la prédominance de certains régimes de l'image symbolique et traduisant de manière consciente une vision particulière de l'individu. Le romancier compose ainsi une grille de lecture permettant d'apprécier son écriture comme régie par divers modes de manipulation de l'imaginaire, étant donné qu'il ne cesse de remodeler les identités de ses personnages en exploitant les ressources sémantiques des sobriquets.

\section{Conclusion}

En somme, les noms qui servent à désigner les personnages d'Alain Mabanckou peuvent donner lieu à d'innombrables commentaires sociologiques et anthropologiques. Ils sont imbus d'une matière informationnelle rendant nécessaire "la distinction du message littéral et du message symbolique" qu'ils véhiculent (Barthes, 1964: 45). Leur distribution se fait selon des choix mûrement réfléchis, afin qu'ils contribuent au mieux au déroulement de l'histoire au cœur de laquelle se trouvent les personnages qui les portent. Chargés de sens, ils participent d'un exercice de communication et "ont quelque chose de propre par rapport aux autres éléments de la langue et du discours, qui les rapproche de la deixis, cette dimension de la subjectivité inscrite dans le langage" (Fédry, 2009: 102) et dont tout texte littéraire est porteur. Il faut néanmoins indiquer qu'ils reposent essentiellement sur une imagerie d'inspiration populaire. Avec Mabanckou, les noms acquièrent une place de choix dans le récit fictionnel et mettent à l'épreuve la réceptivité du lecteur, surtout dans le cas des sobriquets. Notre sentiment est que ces derniers nous donnent en effet accès au " jardin secret" des personnages, étant entendu qu'ils ont vocation à mieux dévoiler leur rapport au monde sensible. 
Cette approche dans la création littéraire aide à la mise au point d'une œuvre cohérente. Dans les romans d'Alain Mabanckou, les anthroponymes demeurent d'habiles préparations qui définissent les personnages dans l'absolu. C'est justement fort de la résonnance qu'ils peuvent avoir dans tout texte littéraire que Proust préconisait d'appréhender les anthroponymes comme des realia qui génèrent une atmosphère dans laquelle il est plaisant de se plonger (Proust, 1929: 233).

\section{Références bibliographiques}

Achebe, Chinua. 2000. Le monde s'effondre. Paris, Présence Africaine.

BARTHES, Roland. 1964. "Rhétorique de l'images" in Communications, 40-51.

Barthes, Roland. 1972. Nouveaux essais critiques. Paris, Seuil.

BrA, Bosson. 2013. "Motivations onomastiques: esthétique de la laideur morale et singularité romanesque chez Jean-Marie Adiaffi. L'exemple des Naufragés de l'intelligence et La carte d'identité" in Sudlangues, $\mathrm{n}^{\circ}$ 19, 102-116.

BAssintsa-Bouesso, Aetius. 2018. "Imaginaire subsaharien et représentations de la figure du sorcier chez Alain Mabanckou" in Interfrancophonies, $\mathrm{n}^{\circ}$ 9, 57-74.

DevÉZA, Jean-Michel. 2012. "L'Afrique à l'identité sans passé d'Alain Mabanckou" in Afrique contemporaine, $\mathrm{n}^{\circ} 241,93-110$.

FÉDRY, Jacques. 2009. “Le nom, c'est l'homme”, in L'Homme Revue française d'anthropologie, $\mathrm{n}^{\circ}$ 191, 77-106. <https://journals.openedition.org/lhomme/22195> [20/06/2020].

GASQUY-Resh, Yannick. 2001. Écrivains francophones du XXème siècle. Paris, Ellipse / AUF.

Grutman, Rainier. 2002. "Bilinguisme" in Le dictionnaire du littéraire. Paul Aron, Denis Saint-Jacques \& Alain Viala (dir). Paris, PUF. 72-73.

Jouve, Vincent. 1992. L'effet-personnage dans le roman. Paris, PUF.

Jouve, Vincent. 2006. La poétique du roman. Paris, Armand Colin.

Kesteloot, Lilyan. 2004. Histoire de la littérature négro-africaine. Paris, Karthala-AUF.

Lopes, Henri. 1982. Le pleurer-rire. Paris, Présence Africaine.

Mabanckou, Alain. 2002. Les petits-fils nègres de Vercingétorix. Paris, Le Serpent à Plumes.

Mabanckou, Alain 2003. African psycho. Paris, Le Serpent à Plumes.

Mabanckou, Alain.2005. Verre cassé. Paris, Seuil.

Mabanckou, Alain. 2006. Mémoires de porc-épic. Paris, Seuil. 
LA FICTION ROMANESQUE COMME RÉCEPTACLE D'ANTHROPONYMES CHARGÉS DE SENS CHEZ...

Mabanckou, Alain. 2007. Lettre à Jimmy. Paris, Fayard.

Mabanckou, Alain. 2012. Tais-toi et meurs. Paris, La Broche.

Mabanckou, Alain. 2012. Le sanglot de l'homme noir. Paris, Fayard.

Mabanckou, Alain. 2013. Lumières de Pointe-Noire. Paris, Seuil.

Marques, Antonio J. S. "Histoire(s) de sobriquets: du populaire au littéraire" $<$ https://cvc. cervantes.es/lengua/paremia/pdf/002/025_souto.pdf $>$ [15/10/2017].

MaYimona, Alphonse. 1992. Le retour triomphal de Mossi. Abidjan, NEA.

Ngoïe-Ngalla, Dominique 1999. Congo-Brazzaville, le retour des ethnies, la violence identitaire. Abidjan, Imprimerie Multiprint.

Ndinga Mво, Abraham. 1988. "Brève histoire du Congo jusqu'à l'arrivée des Français" in Notre Librairie. $n^{\circ}$ 92-93, 1988, 40-43.

N'GANA, Yeo. 2016. "La littérature des noms dans La carte d'identité de Jean-Marie Adiaffi” $<$ https://www.researchgate.net/publication/314207252_La_litterature_des_noms_dans_La carte_d'identite_de_Jean-Marie_Adiaffi > [17/07/2020].

PAVlovic, Diane. 1987. "Du cryptogramme au nom réfléchi. Onomastique ducharmienne" in Études françaises. Vol 23, n 3, 89-98. <https://www.erudit.org/fr/revues/etudfr/1987-v23n3-etudfr1058/035729ar.pdf> [14/04/2020].

Porte, Danielle. 2013 Vercingétorix: celui qui fit trembler César. Paris, Ellipse Marketing.

Proust, Marcel. 1929. Du côté de chez Swan. Paris, Gallimard.

TABa Odounga, Didier. 2013. "La langue française comme vecteur des savoirs et d'axiologie chez Alain Mabanckou" in Regards sur la langue française au Congo. Anatole Mbanga (dir). Paris, L'Harmattan.

TREPS, Marie. 2012. Le roman de la gloire: les sobriquets des politiques. Paris, Seuil. 